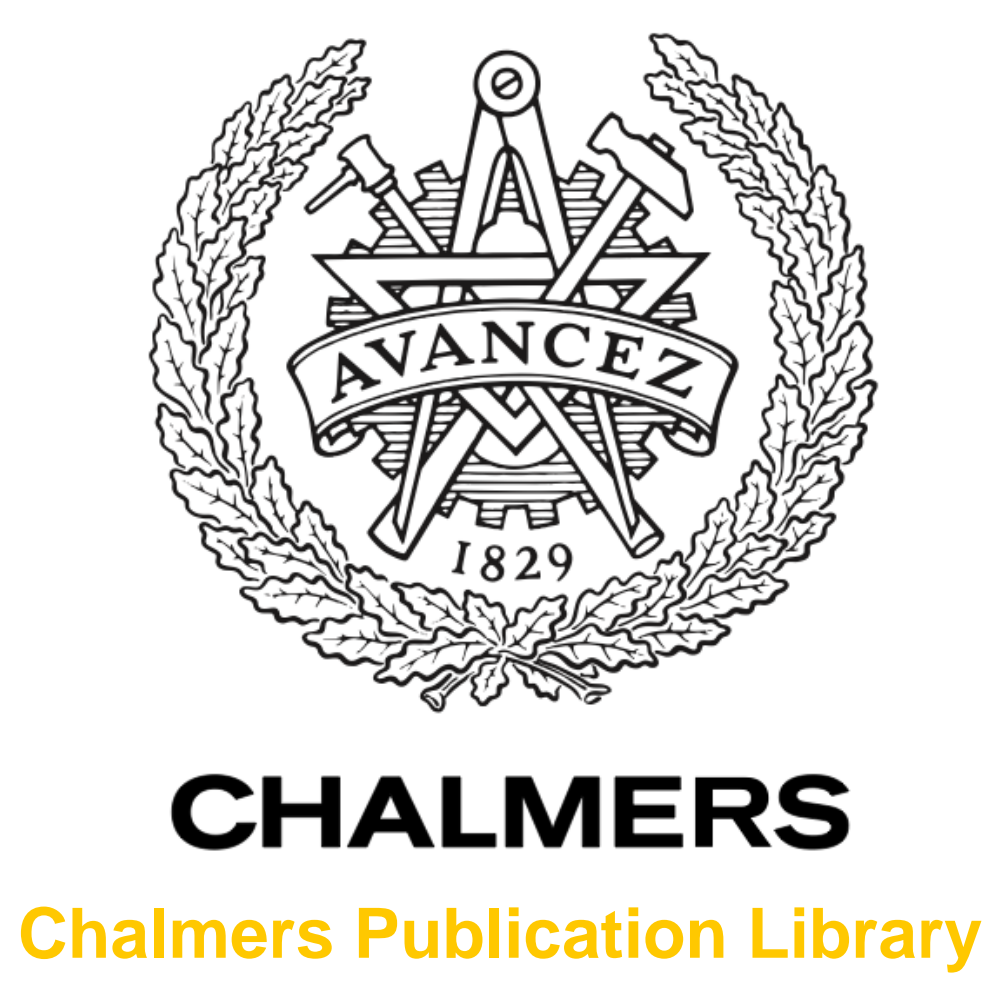

\title{
Origin of resolution enhancement by co-doping of scintillators: Insight from electronic structure calculations
}

This document has been downloaded from Chalmers Publication Library $(\mathrm{CPL})$. It is the author's version of a work that was accepted for publication in:

\section{Applied Physics Letters (ISSN: 0003-6951)}

Citation for the published paper:

Aberg, D. ; Sadigh, B. ; Schleife, A. (2014) "Origin of resolution enhancement by co-doping of scintillators: Insight from electronic structure calculations". Applied Physics Letters, vol. 104(21),

http://dx.doi.org/10.1063/1.4880576

Downloaded from: http://publications.lib.chalmers.se/publication/200392

Notice: Changes introduced as a result of publishing processes such as copy-editing and formatting may not be reflected in this document. For a definitive version of this work, please refer to the published source. Please note that access to the published version might require a subscription. 


\title{
Origin of resolution enhancement by co-doping of scintillators: Insight from electronic structure calculations
}

\author{
Daniel Åberg, ${ }^{1, a)}$ Babak Sadigh, ${ }^{1}$ André Schleife, ${ }^{2}$ and Paul Erhart $\left.{ }^{3, b}\right)$ \\ ${ }^{1}$ Physical and Life Sciences Directorate, Lawrence Livermore National Laboratory, Livermore, \\ California 94550, USA \\ ${ }^{2}$ Department of Materials Science and Engineering, University of Illinois at Urbana-Champaign, \\ Urbana, Illinois 61801, USA \\ ${ }^{3}$ Department of Applied Physics, Chalmers University of Technology, Gothenburg, Sweden
}

(Received 25 April 2014; accepted 13 May 2014; published online 29 May 2014)

\begin{abstract}
It was recently shown that the energy resolution of Ce-doped $\mathrm{LaBr}_{3}$ scintillator radiation detectors can be crucially improved by co-doping with $\mathrm{Sr}, \mathrm{Ca}$, or $\mathrm{Ba}$. Here, we outline a mechanism for this enhancement on the basis of electronic structure calculations. We show that (i) $\mathrm{Br}$ vacancies are the primary electron traps during the initial stage of thermalization of hot carriers, prior to hole capture by Ce dopants; (ii) isolated $\mathrm{Br}$ vacancies are associated with deep levels; (iii) Sr doping increases the $\mathrm{Br}$ vacancy concentration by several orders of magnitude; (iv) $\mathrm{Sr}_{\mathrm{La}}$ binds to $V_{\mathrm{Br}}$ resulting in a stable neutral complex; and (v) association with $\mathrm{Sr}$ causes the deep vacancy level to move toward the conduction band edge. The latter is essential for reducing the effective carrier density available for Auger quenching during thermalization of hot carriers. Subsequent de-trapping of electrons from $\mathrm{Sr}_{\mathrm{La}}-V_{\mathrm{Br}}$ complexes can activate Ce dopants that have previously captured a hole leading to luminescence. This mechanism implies an overall reduction of Auger quenching of free carriers, which is expected to improve the linearity of the photon light yield with respect to the energy of incident electron or photon. (c) 2014 AIP Publishing LLC. [http://dx.doi.org/10.1063/1.4880576]
\end{abstract}

Scintillator radiation detectors have many applications in nuclear and radiological surveillance, high-energy physics, and medical imaging. ${ }^{1-3}$ The energy resolved detection of radiation is of particular interest as it enables, for example, the identification of fissile materials. ${ }^{4}$ According to counting statistics, the resolution increases with luminosity, which usually results from a higher conversion efficiency, i.e., relatively more photons are generated per incident energy. In practice, the resolution is further limited by the non-linear response of the scintillator to the energy of the incident radiation. ${ }^{5}$ It is usually accepted that the resulting non-proportionality arises from the competition between non-radiative quenching, defect carrier trapping, as well as activator capture and subsequent emission. ${ }^{6-10}$

One of the most promising materials for detector performance is Ce-doped $\mathrm{LaBr}_{3}{ }^{11}$ It yields an energy resolution of $2.7 \%$ at a photon energy of $662 \mathrm{keV}$ in combination with an extremely fast scintillation pulse. $\mathrm{LaBr}_{3}$ has been very well characterized both experimentally ${ }^{12}$ and theoretically. ${ }^{13-15}$ The prospect of improving energy resolution by co-doping $\mathrm{LaBr}_{3}$ :Ce with $\mathrm{Sr}$ or $\mathrm{Ba}$ was first noted experimentally by Yang et al. ${ }^{16}$ Later, Alekhin et al. revisited this aspect and using $\mathrm{Ca}$ and $\mathrm{Sr}$ achieved an improvement of energy resolution down to $2.0 \%$ at $662 \mathrm{keV} .{ }^{17} \mathrm{~A}$ more comprehensive investigation, including both the alkaline as well as earth-alkaline series, revealed that better performance is only achievable when using the heavier elements of the latter series $(\mathrm{Sr}, \mathrm{Ca}$, and $\mathrm{Ba}){ }^{18,19}$ Several possible mechanisms were tentatively proposed to rationalize

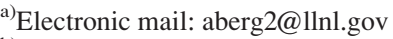

b)Electronic mail: erhart@chalmers.se
}

these observations: ${ }^{20}$ (i) reduction of the nonradiative recombination rate, (ii) an increase of the so-called escape rate of the carriers from the quenching region, or (iii) an increase in the trapping rate of $\mathrm{Ce}^{3+}$. Here, we address this question via first principles calculations of thermodynamic and electronic properties of intrinsic and extrinsic defects as well as their complexes in $\mathrm{Ce}$ and $\mathrm{Sr}$-doped $\mathrm{LaBr}_{3}$.

Calculations were performed within density functional theory (DFT) using the projector augmented wave (PAW) method, ${ }^{21}$ as implemented in the Vienna ab-initio simulation package. ${ }^{22}$ Exchange-correlation was treated within the generalized gradient approximation (GGA). ${ }^{23} \mathrm{DFT}+U$ type on-site potentials ${ }^{24}$ were included for both La- $4 f$ $\left(U_{\text {eff }}=10.3 \mathrm{eV}\right)$ and Ce- $4 f$ states $\left(U_{\text {eff }}=1.2 \mathrm{eV}\right)$ in order to obtain the correct ordering of La-5d and $4 f$ states and to reproduce experimental Ce- $4 f$ ionization energies. ${ }^{14,15,25}$ The plane-wave energy cutoff was set to $230 \mathrm{eV}$ and Gaussian smearing with a width of $0.1 \mathrm{eV}$ was used to determine the occupation numbers. Excited Ce- $4 f^{0}$ states were obtained in a similar, albeit more flexible, and automatized approach, as used by Canning and co-workers. ${ }^{14}$ First, a subspace of Ce- $4 f$ states is determined by projection of single particle wave functions on spherical harmonics within the PAW spheres, which is possible for localized atomic states such as the rare-earth $4 f$ states. The $4 f$ occupation number can then be controlled by introducing a separate electron chemical potential for this subspace. Hole polarons were studied using the polaron self-interaction correction method (pSIC), see Ref. 26.

Lanthanum bromide adopts a hexagonal lattice structure in space group $176\left(\mathrm{P}_{3} / \mathrm{m}\right)$ with $\mathrm{La}$ and $\mathrm{Br}$ ions occupying Wyckoff sites $2 c$ and $6 h$, respectively. The calculated lattice 
parameters are $a=8.140 \AA$ and $c=4.565 \AA$ to be compared with experimental values of $a=7.9648(5) \AA$ and $c=4.5119(5) \AA$, respectively, see Fig. $1 .^{27}$ Defects were modeled using 168 -atom supercells. $\Gamma$-point sampling was found to be sufficient to converge defect formation energies to better than $0.05 \mathrm{eV}$. Configurations were relaxed until ionic forces were less than $10 \mathrm{meV} / \AA$. Defect formation energies were calculated using the formalism described in Ref. 28. Potential alignment as well as periodic image charge corrections were taken into account to correct for finite size effects, ${ }^{29,30}$ and band gap corrections were applied to adjust for the underestimation of the band gap. ${ }^{30,31}$ Band edge offsets were determined by $G_{0} W_{0}$ calculations as described in Ref. 15. The thus obtained band gap of $5.3 \mathrm{eV}$ is much closer to the experimental value of $5.9 \mathrm{eV}$ than the $\mathrm{DFT}+U$ value of $3.6 \mathrm{eV}$. We estimate that transition levels are associated with an error of $0.1-0.2 \mathrm{eV}$ due to the approximate exchange-correlation functional. Defect concentrations were obtained using the calculated formation energies on the basis of a self-consistent solution of the charge neutrality condition. $^{28,32}$

In agreement with the earlier calculations, ${ }^{14}$ we find that in its ground state Ce preferentially substitutes for La with small distortions and adopts a neutral charge state corresponding to a $\mathrm{Ce}^{3+}-4 f^{1} 5 d^{0}$ configuration. By choice of the $\mathrm{DFT}+U$ parameters, the occupied $4 f$ level is located $0.9 \mathrm{eV}$ above the valence band maximum (VBM). The excited Ce- $4 f^{0} 5 d^{1}$ state, which is obtained by enforcing the deoccupation of Ce- $4 f$ levels, is associated with the emergence of electronic levels very close to the conduction band minimum (CBM). They are predominantly of $\mathrm{Ce}-5 d$ character and strongly hybridized with the neighboring La-5d states.

From an extensive exploration of intrinsic defects including polaronic configurations, one obtains bromine vacancies $V_{\mathrm{Br}}$ as the energetically most favorable donor-type defect under both $\mathrm{La}$ and Br-rich conditions, see Fig. 2(a). The formation energy of the $\mathrm{Br}$ vacancy at the VBM varies between $-3.7 \mathrm{eV}$ and $-0.9 \mathrm{eV}$ corresponding to $\mathrm{La}$ and $\mathrm{Br}$-rich conditions, respectively. $\mathrm{Br}$ vacancy formation is, therefore, thermodynamically more favorable than formation of self-trapped holes $\left(V_{K}\right.$-centers), for which a formation energy of $-0.3 \mathrm{eV}$ is obtained irrespective of chemical boundary conditions. The $\mathrm{Br}$ vacancy is associated with an equilibrium transition level $(+1 / 0) 0.55 \mathrm{eV}$ below the CBM, (a)

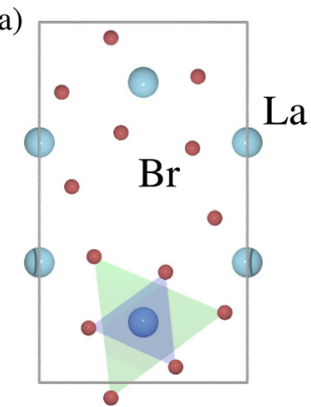

(b)

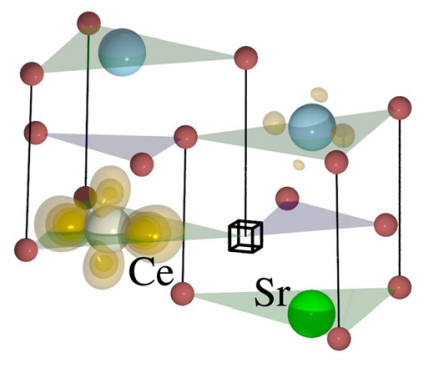

FIG. 1. (a) View along $z$-axis of the conventional $\mathrm{LaBr}_{3}$ cell. (b) Example of a strontium-vacancy-cerium defect cluster $\left(\mathrm{Sr}_{\mathrm{La}}-V_{\mathrm{Br}}\right)-\mathrm{Ce}_{\mathrm{La}}$. The vacancy is illustrated by the hollow black cube. The charge density associated with the electron trap level is visualized by the yellow isosurfaces, showing a clear Ce-5d component.

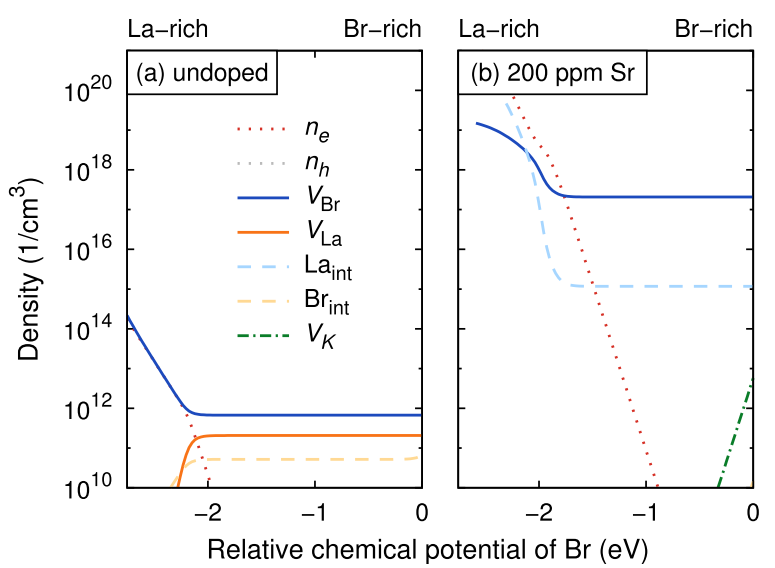

FIG. 2. Equilibrium defect and charge carrier concentrations as a function of the $\mathrm{Br}$ chemical potential at a temperature of $600 \mathrm{~K}$. Results are shown both for (a) pure material and (b) $\mathrm{LaBr}_{3}$ doped with $200 \mathrm{ppm} \mathrm{Sr}$ corresponding to the experimental doping conditions. Note that doping with $\mathrm{Sr}$ increases the $\mathrm{Br}$ vacancy concentration by several orders of magnitude. (Ce was not explicitly included in these figures, since it does not affect the charge neutrality condition.)

see Fig. 3, and a trap state $0.3 \mathrm{eV}$ below the CBM. The associated single particle level is located inside the band gap and has La-5d character. It can thus act as an efficient electron trap, effectively removing carriers from the light-generation process during the instrumentation pulse shape-time.

The inclusion of strontium is accomplished by substitution on lanthanum sites. The resulting $\mathrm{Sr}_{\mathrm{La}}^{\prime}$ defect acts as a shallow acceptor with a vanishingly small lattice distortion. Assuming a $\mathrm{Sr}$ concentration of $200 \mathrm{ppm}$, the concentration of $\mathrm{Br}$ vacancies will increase by several orders of magnitude compared to pristine or Ce-only doped material as shown in Fig. 2. The opposite charge states of $V_{\mathrm{Br}}^{\cdot}$ and $\mathrm{Sr}_{\mathrm{La}}^{\prime}$ cause a mutual attraction, which is quantified in Fig. 4 revealing a binding energy of $-0.3 \mathrm{eV}$ for the nearest neighbor $\left(\mathrm{Sr}_{\mathrm{La}}-V_{\mathrm{Br}}\right)^{\times}$complex. ${ }^{33}$ See Fig. 1(b) for an example of complex geometry. A closer inspection of the electronic structure of the complex reveals that both trap and equilibrium transition levels, which are associated with the $\mathrm{Br}$ vacancy, shift closer to the CBM by approximately $0.2 \mathrm{eV}$ compared to the isolated vacancy, see Fig. 3.

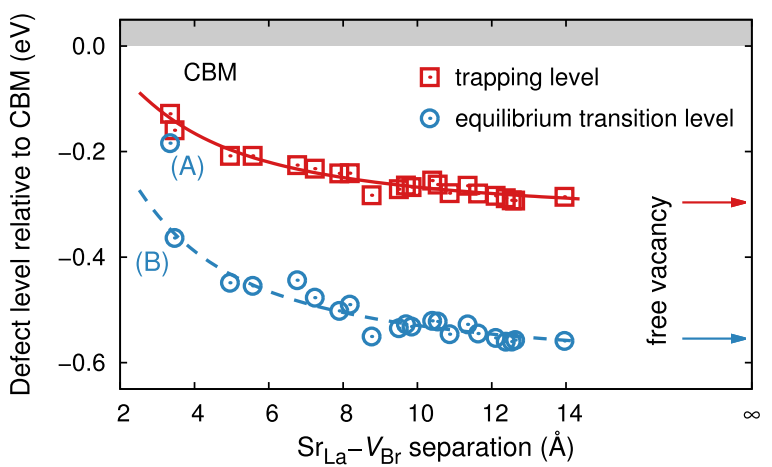

FIG. 3. Trapping and equilibrium transition levels for the $(+1 / 0)$ transition of $\mathrm{Sr}_{\mathrm{La}}-V_{\mathrm{Br}}$ as a function of $\mathrm{Sr}_{\mathrm{La}}-V_{\mathrm{Br}}$ separation. The former was calculated by considering the transition level for fixed ionic positions starting from the $V_{\mathrm{Br}}^{\cdot}$ configuration. The equilibrium transition level, on the other hand, was computed allowing full relaxation in both charge states. The data points labelled A and B indicate out-of-plane (A) and in-plane (B) configurations of nearest neighbor defect complexes, compare Fig. 1. 


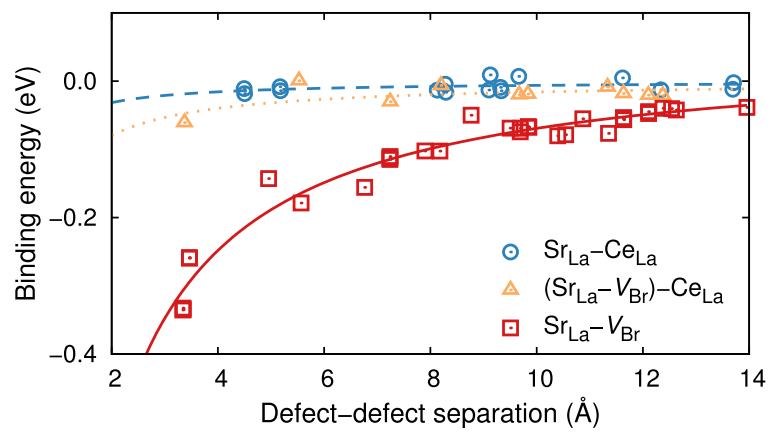

FIG. 4. Binding energy as a function of defect separation for various defect associates. While there is a strong attraction between $V_{\mathrm{Br}}^{\cdot}$ and $\mathrm{Sr}_{\mathrm{La}}^{\prime}$, the interaction of $\mathrm{Ce}_{\mathrm{La}}^{\times}$with other defects is weak. The variation in the binding energies is related to the anisotropy of the crystal structure.

The shift of the defect level can be rationalized by considering that $\mathrm{Sr}_{\mathrm{La}}^{\prime}$ introduces a point charge-like electrostatic potential that shifts the local energy scale. Localized states, such as the $V_{\mathrm{Br}}$ defect level, are sensitive to this shift, whereas the delocalized states that make up the valence and conduction bands are unaffected, causing an effective upward shift of the vacancy level.

We can now consider the effect of the $\mathrm{Sr}_{\mathrm{La}}-V_{\mathrm{Br}}$ complex on the electronic structure of $\mathrm{Ce}_{\mathrm{La}}$. Even though the binding between $\mathrm{Ce}_{\mathrm{La}}$ and $\mathrm{Sr}_{\mathrm{La}}-V_{\mathrm{Br}}$ is weak as shown in Fig. 4, the large concentration of $\mathrm{Ce}(5 \%)$ used experimentally ${ }^{16,17}$ implies that the average separation between $\mathrm{Ce}_{\mathrm{La}}$ and $\mathrm{Sr}_{\mathrm{La}}-V_{\mathrm{Br}}$ is only about 2.5 unit cells. As a result, each $\mathrm{Sr}_{\mathrm{La}}-V_{\mathrm{Br}}$ complex will have a $\mathrm{Ce}$ atom in its vicinity. One can thus expect the spectroscopic signatures of cerium in Sr-doped $\mathrm{LaBr}_{3}$ :Ce to be affected. To demonstrate this effect we have calculated $\mathrm{Ce}(4 f \leftrightarrow 5 d)$ excitation and emission energies for isolated $\mathrm{Ce}_{\mathrm{La}}$ as well for various complexes of $\mathrm{Ce}_{\mathrm{La}}$ with $\mathrm{Sr}_{\mathrm{La}}-V_{\mathrm{Br}}$, where the latter are nearest neighbors. Spin-orbit interaction was not included self-consistently but rather added as a perturbation to the $4 f$ states according to

$$
\Delta E_{\mathrm{so}}= \begin{cases}-2 \xi_{4 f} & j=\frac{5}{2} \\ \frac{3}{2} \xi_{4 f} & j=\frac{7}{2},\end{cases}
$$

where $\xi_{4 f}=0.1 \mathrm{eV}$ was obtained from the $4 f$ splitting in a Ce- $4 f^{0} 5 d^{1}$ configuration.

The thus obtained optical signatures can be categorized as follows (also compare Table I): Isolated $\mathrm{Ce}$ is associated with the largest excitation energy and a substantial Stokes shift. The magnitude of the Stokes shift is in agreement with the earlier calculations by Andriessen et al. ${ }^{34}$ For configurations, in which the $\mathrm{Br}$ vacancy is a first neighbor of $\mathrm{Ce}$, a pronounced reduction of excitation energies is observed with a typical value of $-0.32 \mathrm{eV}$. Due to small lattice relaxations in the excited state, the emission energies are not strongly reduced. As a result, these configurations are associated with a smaller Stokes shift $(0.16 \mathrm{eV})$ than the isolated Ce site. There are several configurations with similar optical signatures. Given the accuracy that can be expected from the present DFT calculations, however, we abstain from a more detailed differentiation of these complexes and in Table I
TABLE I. Comparison of calculated and experimental data for Ce excitation and emission $\left(4 f^{1} 5 d^{0} \leftrightarrow 4 f^{0} 5 d^{1}\right)$. Values in parentheses in the excitation column indicate the shift with respect to free $\mathrm{Ce}_{\mathrm{La}}$, which is identical to site I in the case of the experimental data. Two values are given in the emission column corresponding to final states of ${ }^{2} F_{5 / 2}$ and ${ }^{2} F_{7 / 2}$, respectively. Experimental data are taken from Ref. 20. Note that the band gap error of DFT manifests itself in a systematic underestimation of all excitation and emission energies. Within the supercell approximation and the GGA exchange correlation functional, the error associated with excitation and emission energies is on the order of $0.05 \mathrm{eV}$.

\begin{tabular}{llcc}
\hline \hline Site & $\begin{array}{c}\text { Excitation } \\
(\mathrm{eV})\end{array}$ & $\begin{array}{c}\text { Emission } \\
(\mathrm{eV})\end{array}$ & $\begin{array}{c}\text { Stokes } \\
\text { shift }(\mathrm{eV})\end{array}$ \\
\hline Calculation & & & \\
$\mathrm{Ce}_{\mathrm{La}}$ & 3.56 & $3.13 / 2.78$ & 0.43 \\
$\left(\mathrm{Sr}_{\mathrm{La}}-V_{\mathrm{Br}}\right)-\mathrm{Ce} \mathrm{La}_{\mathrm{La}}$ & $3.24(-0.32)$ & $3.08 / 2.73$ & 0.16 \\
Experiment (Ref. 20) & & & \\
I & 4.03 & $3.47 / 3.19$ & 0.56 \\
$\mathrm{II}$ & $3.59(-0.44)$ & $3.36 / 3.10$ & 0.24 \\
III & $3.47(-0.56)$ & $3.27 / 3.10$ & 0.21 \\
\hline \hline
\end{tabular}

only include the results for a representative cluster. Finally, configurations, for which $\mathrm{Ce}$ and $V_{\mathrm{Br}}$ are not first nearest neighbors, behave similar to isolated $\mathrm{Ce}$.

We now wish to point out several important facts. First, we note that the shift in the calculated excitation energy $(0.34 \mathrm{eV})$ between $\mathrm{Ce}_{\mathrm{La}}$ and $\left(\mathrm{Sr}_{\mathrm{La}}-V_{\mathrm{Br}}\right)-\mathrm{Ce}_{\mathrm{La}}$ is close to the experimental shift between sites I and II/III $(0.44 / 0.56 \mathrm{eV})$. Thus, we associate site I with $\mathrm{Ce}_{\mathrm{La}}$ and II/III with nearest neighbor triple complexes. This identification is further corroborated by the agreement between calculated and experimental Stokes shifts for $\mathrm{Ce}_{\mathrm{La}}(0.43 \mathrm{eV})$ and site I $(0.56 \mathrm{eV})$, as well as for $\left(\mathrm{Sr}_{\mathrm{La}}-V_{\mathrm{Br}}\right)-\mathrm{Ce}_{\mathrm{La}}(0.16 \mathrm{eV})$ and sites II/III $(0.24 / 0.21 \mathrm{eV})$.

Since $\mathrm{Ce}$ is a nearest neighbor of a $\mathrm{Br}$ vacancy in these configurations, the deep trap level associated with the vacancy will, to a large part, consist of Ce-5d states, see Fig. 1. This explains the reduction of the excitation energy for this complex compared to isolated $\mathrm{Ce}_{\mathrm{La}}$. Furthermore, if we assume the $4 f$ level to be unaffected by the neutral complex $\mathrm{Sr}_{\mathrm{La}}-V_{\mathrm{Br}}$, it implies that we can identify the shift in excitation energy with trap depth.

To summarize, we demonstrated that co-doping of $\mathrm{LaBr}_{3}$ :Ce with strontium gives rise to a shallow acceptor substituting on a lanthanum site. Overall charge neutrality requires formation of one oppositely charged bromine vacancy for each strontium atom, resulting in a steep increase of Br vacancy concentration compared to the undoped material. Moreover, the two defects are electrostatically attracted to each other and will form stable complexes that act as electron traps. Upon complexation, the vacancy defect level moves closer to the CBM, becoming more shallow. Complexes in close proximity to $\mathrm{Ce}$ activators will display distinct optical signatures in close correspondence to recent measurements. ${ }^{20}$ The occupied Ce $4 f$ level is located rather deep inside the gap $(0.6-0.9 \mathrm{eV})$ and will, therefore, have a low but finite hole capture rate. If we assume that $\mathrm{Ce}$ is activated by sequential capture of hole and electron, this implies that hole capture is the rate limiting step. Since Auger recombination, which has been shown to be the major quenching channel at this time scale for halide scintillators, ${ }^{35}$ 
has a cubic dependence on the electron and hole densities, the carrier population will be greatly reduced by non-linear quenching causing an overall non-proportional response. If, however, $\mathrm{Sr}_{\mathrm{La}}-V_{\mathrm{Br}}$ traps are active during the initial thermalization stage (2-10 ps in halide systems) they will effectively reduce the free electron density. As a result, a larger density of holes will remain available for ionization of cerium activators without being quenched by the Auger mechanism. Although calculation of electron capture cross-sections for the complexes is beyond the scope of this Letter, we note that a very fast capture is indeed possible. For example, in picosecond optical absorption experiments, it is shown that energy transfer to europium activators in $\mathrm{SrI}_{2}$ : Eu may be as fast as $400 \mathrm{fs}^{36}$

Another time-scale of importance is the de-trapping rate from the Br-vacancy sites. As alluded to earlier, each defect complex will be in close proximity to a $\mathrm{Ce}$ atom. Once any of the nearby $\mathrm{Ce}$ atoms captures a hole, Coulombic attraction serves as a driving force for transferring the electron from the complex to the activator. This suggests that non-linear quenching is reduced at the cost of longer decay-times; in fact, two of the three cerium sites discussed by Alehkin are associated with very long decay times ranging from $60-2500 \mathrm{~ns}$ while accounting for $20 \%-45 \%$ of the total light output. $^{20}$

We acknowledge fruitful discussions with S. Payne, G. Bizarri, and R. T. Williams. This work was performed under the auspices of the U.S. Department of Energy by Lawrence Livermore National Laboratory under Contract No. DEAC52-07NA27344 with the support from the National Nuclear Security Administration Office of Nonproliferation Research and Development (NA-22). P.E. acknowledges funding from the Area of Advance-Materials Science at Chalmers and the Swedish Research Council (Vetenskapsraidet). Computer time allocations by the Swedish National Infrastructure for Computing at NSC (Linköping) and C3SE (Gothenburg) are gratefully acknowledged.

${ }^{1}$ P. A. Rodnyi, Physical Processes in Inorganic Scintillators (CRC Press, Boca Raton, 1997).

${ }^{2}$ G. F. Knoll, Radiation Detection and Measurement, 4th ed. (Wiley, New York, NY, 2010).

${ }^{3}$ M. Nikl, A. Vedda, and V. V. Laguta, in Springer Handbook of Crystal Growth, edited by G. Dhanaraj, K. Byrappa, V. Prasad, and M. Dudley (Springer, Berlin, Heidelberg, 2010), pp. 1663-1700.

${ }^{4}$ K. E. Nelson, T. B. Gosnell, and D. A. Knapp, Nucl. Instrum. Methods Phys. Res., Sect. A 659, 207 (2011)

${ }^{5}$ P. Dorenbos, IEEE Trans. Nucl. Sci. 57, 1162 (2010)

${ }^{6}$ P. Dorenbos, Phys. Status Solidi A 202, 195 (2005).

${ }^{7}$ A. V. Vasil'ev, IEEE Trans. Nucl. Sci. 55, 1054 (2008).
${ }^{8}$ S. Kerisit, K. M. Rosso, B. D. Cannon, F. Gao, and Y. Xie, J. Appl. Phys. $\mathbf{1 0 5}, 114915$ (2009).

${ }^{9}$ G. Bizarri, W. Moses, J. Singh, A. Vasil'ev, and R. Williams, J. Lumin. 129, 1790 (2009).

${ }^{10}$ S. Payne, W. W. Moses, S. Sheets, L. Ahle, N. Cherepy, B. Sturm, S. Dazeley, G. Bizarri, and W.-S. Choong, IEEE Trans. Nucl. Sci. 58, 3392 (2011).

${ }^{11}$ E. V. D. van Loef, P. Dorenbos, C. W. E. van Eijk, K. Krämer, and H. U. Güdel, Appl. Phys. Lett. 79, 1573 (2001).

${ }^{12}$ P. Dorenbos, E. V. D. van Loef, A. P. Vink, E. van der Kolk, C. W. E. van Eijk, K. W. Krämer, H. U. Güdel, W. M. Higgins, and K. S. Shah, J. Lumin. 117, 147 (2006); F. P. Doty, D. McGregor, M. Harrison, K. Findley, and R. Polichar, Proc. SPIE 6707, 670705 (2007).

${ }^{13}$ G. Bizarri and P. Dorenbos, Phys. Rev. B 75, 184302 (2007); D. J. Singh, ibid. 82, 155145 (2010).

${ }^{14}$ A. Canning, A. Chaudhry, R. Boutchko, and N. Grønbech-Jensen, Phys. Rev. B 83, 125115 (2011).

${ }^{15}$ D. Åberg, B. Sadigh, and P. Erhart, Phys. Rev. B 85, 125134 (2012).

${ }^{16} \mathrm{~K}$. Yang, P. Menge, J. Buzniak, and V. Ouspenski, in IEEE Nuclear Science Symposium and Medical Imaging Conference (NSS/MIC) (2012), pp. 308-311.

${ }^{17}$ M. S. Alekhin, J. T. M. de Haas, I. V. Khodyuk, K. W. Krämer, P. R. Menge, V. Ouspenski, and P. Dorenbos, Appl. Phys. Lett. 102, 161915 (2013).

${ }^{18}$ P. Dorenbos, M. Alekhin, I. V. Khodyuk, J. T. M. de Haas, and K. Krämer, paper presented at SCINT, Shanghai, China, 2013.

${ }^{19}$ M. S. Alekhin, D. A. Biner, K. W. Krämer, and P. Dorenbos, J. Appl. Phys. 113, 224904 (2013).

${ }^{20}$ M. S. Alekhin, S. Weber, K. W. Krämer, and P. Dorenbos, J. Lumin. 145, 518 (2014).

${ }^{21}$ P. E. Blöchl, Phys. Rev. B 50, 17953 (1994); G. Kresse and D. Joubert, ibid. 59, 1758 (1999).

${ }^{22}$ G. Kresse and J. Hafner, Phys. Rev. B 47, 558 (1993); 49, 14251 (1994); G. Kresse and J. Furthmüller, ibid. 54, 11169 (1996); Comput. Mater. Sci. 6, 15 (1996).

${ }^{23}$ J. P. Perdew, K. Burke, and M. Ernzerhof, Phys. Rev. Lett. 77, 3865 (1996); Erratum, ibid. 78, 1396(E) (1997).

${ }^{24}$ S. L. Dudarev, G. A. Botton, S. Y. Savrasov, C. J. Humphreys, and A. P. Sutton, Phys. Rev. B 57, 1505 (1998).

${ }^{25}$ M. T. Czyżyk and G. A. Sawatzky, Phys. Rev. B 49, 14211 (1994).

${ }^{26}$ B. Sadigh, P. Erhart, and D. Åberg, "A variational polaron self-interaction corrected total-energy functional for charge excitations in wide-band gap insulators," e-print arXiv:1401.7137.

${ }^{27}$ K. Krämer, T. Schleid, M. Schulze, W. Urland, and G. Meyer, Z. Anorg. Allg. Chem. 575, 61 (1989).

${ }^{28}$ S. B. Zhang and J. E. Northrup, Phys. Rev. Lett. 67, 2339 (1991); P. Erhart, D. Åberg, and V. Lordi, Phys. Rev. B 81, 195216 (2010).

${ }^{29}$ G. Makov and M. C. Payne, Phys. Rev. B 51, 4014 (1995).

${ }^{30}$ S. Lany and A. Zunger, Phys. Rev. B 78, 235104 (2008).

${ }^{31}$ D. Åberg, P. Erhart, A. J. Williamson, and V. Lordi, Phys. Rev. B 77, 165206 (2008).

${ }^{32}$ P. Erhart and K. Albe, J. Appl. Phys. 104, 044315 (2008).

${ }^{33} \mathrm{We}$ here adopt the convention that negative binding energies indicate attraction.

${ }^{34}$ J. Andriessen, E. van der Kolk, and P. Dorenbos, Phys. Rev. B 76, 075124 (2007).

${ }^{35}$ J. Q. Grim, K. B. Ucer, A. Burger, P. Bhattacharya, E. Tupitsyn, E. Rowe, V. M. Buliga, L. Trefilova, A. Gektin, G. A. Bizarri, W. W. Moses, and R. T. Williams, Phys. Rev. B 87, 125117 (2013).

${ }^{36}$ K. B. Ucer, G. Bizarri, A. Burger, A. Gektin, L. Trefilova, and R. T. Williams, Phys. Rev. B 89, 165112 (2014). 